\title{
Transmission Power Control Management for Radio PHY802.15.4 based on LQI for Wireless Sensor Network
}

\author{
Neeraj Tantubay \\ School of Information Technology \\ Rajiv Gandhi Technical University \\ Bhopal, India
}

\author{
Sanjeev Sharma \\ School of Information Technology \\ Rajiv Gandhi Technical University \\ Bhopal, India
}

\begin{abstract}
Energy-efficiency is an important design consideration of communication schemes for wireless sensor networks (WSNs), because each wireless sensor device (sensor Node) operates with limited sources of Energy (power). There are mainly three modes of power consumption in communication system: Transmission Mode, Receive Mode and Idle Mode. Energy of nodes is consumed according to transmission power required by the radios. In this research, we devise a mechanism, Transmission Power Control Management (TPCM) that saves the battery power of nodes by minimizing the required transmission power consumption of radio during data packet transmission based on Link Quality Indicator (LQI). IEEE 802.15.4 is a standard that specifies the physical layer and media access control designed for low-rate wireless personal area networks (LR-WPANs), it is used as radio at PHY layer (PHY802.15.4) and protocol at MAC layer (MAC802.15.4), and it is generally used for WSNs. In this paper, we use LQI as a parameter to dynamically set required transmission power of radio.
\end{abstract}

\section{Keywords}

WSN, IEEE 802.15.4 standard, Radio, Transmission Power LQI, TPCM,

\section{INTRODUCTION}

Wireless sensor network (WSN) [1][8] consists of a number of in-situ battery-powered sensor nodes, which communicate through multi-hop or direct paths. A WSN can be employed to collect the data about physical phenomena of interest. There is a large collection of potential applications, including environmental monitoring, object tracking, surveillance, etc. [1][2]. Although WSNs have diverse task-specific requirements, many of them rely on communication scheme among nodes to transmit of data packet of information that is collected by these sensor nodes through radios.

In general, the battery in a sensor node is limited and not rechargeable [3][4][8]. Since wireless communications consume most of the energy to transmit data packet [9], to minimize such transmission power consumption an efficient scheme is greatly desired.

One important approach to save communication energy consumption of nodes is to control transmission power required by radios. Because battery power of nodes is used as transmission power according to transmission power required by radios. Obviously, setting the wireless transmission power of radios in different levels will result in minimizing transmission power. In this research, we have proposed a scheme TPCM to minimize over all transmission power consumption of radio as well as nodes based on LQI. The PHY802.15.4 provides value of LQI based on received message signal strength, Interference power and Noise power. The value of LQI shows strength of link among nodes, means, if the value of LQI is greater it means high signal strength and it may be required less transmission power, and if value of LQI is less it means low signal strength and it may be required high transmission power.

Rest of paper consists, overview of IEEE 802.15.4 that defines PHY802.15.4, LQI, IEEE802.15.4 devices and MAC802.15.4 in section 2, proposed work in section 3, section 4 consists of performance matrices used in this work, section 5 consists of simulation information, result and analysis is shown in section 6 , section 7 and 8 consist of conclusion and future work respectively.

\section{OVERVIEW OF IEEE 802.15.4}

The Institute of Electrical and Electronic Engineer (IEEE) finalized the IEEE 802.15.4 standard [15][16][17] in October 2003. The physical and MAC layers have been defined with this standard for low-rate Wireless Personal Area Network (LRWPAN) [18].

Some of the most attractive applications of this standard can be mentioned like, applications in the area of wireless sensor networks, home automation, home networking, connecting devices to a personal computer (PC), home security and so on [1][8]. The low rate data, low cost communication and low power consumption are the key points of this standard which mainly aimed to fulfill the most requirements of low power network applications like wireless sensor networks.

Generally this standard specifies the MAC layer and the physical layer for Low-Rate Wireless Personal Area Network (LR-WPAN) but it is also suitable for sensor networks since the sensor network can be built up from LR-WPAN.

\subsection{IEEE 802.15.4 Physical Layer}

Generally, transmission, modulation and spreading of data happen at this PHY802.15.4 layer [14][15][16]. The physical layer supports three frequency bands: a $2450 \mathrm{MHz}$ band (with 16 channels), a $915 \mathrm{MHz}$ band (with 10 channels) and a 868 $\mathrm{MHz}$ band (1 channel), all using the Direct Sequence Spread Spectrum (DSSS) access mode. The $2450 \mathrm{MHz}$ band employs Offset Quadrature Phase Shift Keying (O-QPSK) for modulation while the $868 / 915 \mathrm{MHz}$ bands rely on Binary Phase Shift Keying (BPSK) [15][16]. 
Some of the key characteristics of the PHY layer [15] are:

-Activation and deactivation of transceiver.

- Estimation of the received signal strength on a given channel (Energy detection(ED)).

- Computing Link quality (LQI)

- Sending and receiving data throughout the available radio channel (the different radio frequencies).

\subsubsection{Link Quality Indicator}

Using CC2420 evaluate the measurement of LQI [12][13][14][15]. CC2420 operates in $2.4 \mathrm{GHz}$ ISM band with an effective data rate of $256 \mathrm{kbps}$, a much higher rate than older radios [13][14]. In the $2.4 \mathrm{GHz}$ band, it has 16 channels with each channel occupying a $3 \mathrm{MHz}$ bandwidth with a center frequency separation of $5 \mathrm{MHz}$ for adjacent channels. CC2420 uses an encoding scheme that encodes 32 chips for a symbol of 4 bits. This encoded data is then OQPSK modulated. CC2420 provides two useful measurements: RSSI and LQI [14][15].

In IEEE 802.15.4 standard [13] [14][15], the LQI measurement is defined as a characterization of the strength and/or quality reception of a packet. The value of the LQI is calculated at physical layer. The LQI measurement is performed for each received packet, and the result is reported to the MAC sublayer as an integer ranging from 0 to 255 . The minimum and maximum LQI values ( 0 and 255$)$ are associated with the lowest and the highest signal qualities detectable by the receiver and the values of LQI is always varies between these two limits. LQI can be formulated [19][20] as:

$$
L Q I=\frac{\text { Receive Msg Power }}{\text { (Interference Power }+ \text { Noise Power) }}
$$

Where,

Receive Msg Power shows the value of signal strength of received signal. Interference power gives the value of interference of coming signal to other signal on the same radio and noise power is a value of noise in that environment. By such values the definition of LQI can be summarized as: LQI is a ratio of received signal strength to sum of Interference power and noise power.

\subsection{IEEE 802.15.4 Devices}

There are two types of devices for which IEEE 802.15.4 standard supports [1][7][10][20], FFD and RFD. These devices contain different functionality and consumed different amount of energy.

(A) Full Function Device (FFD):- It can initialize and manage a network [20]. It can also associate with the other devices in the network. FFD can be used in all network topologies. A FFD can support three operational modes:

- A Personal Area Network (PAN) coordinator, which uses for identifying a network.

- A coordinator who associates with the other devices in the network.

- A simple device which has just the simple functionality for sensing and collecting data.
A FFD can communicate with all other devices regardless that they are FFD or RFD. Any sensor network needs at least one FFD device for coordinating the network. It has high power consumption and performs different functionality in networks.

(B) Reduced Function Device (RFD):- A RFD device is operated with minimal implementation ability, only for simple operations [20]. It does not have the need to send large amounts of data and it communicates only with a single FFD at a certain time. It can just send and receive data from other devices in network. A RFD device is battery operated with a small RAM. The most end-point nodes in the network are RFD. They use low energy for only sensing and collect data from an environment and communication with a FFD.

\subsection{IEEE 802.15.4 Medium Access Control (MAC) Layer}

This layer works like an interface between the Physical layer and the Network layer [15][16]. The main feature of this layer is the reducing the probability for data collision. MAC layer [13][14] uses a LQI function for seeking a free channel for data transmission. The data transmission [15] happens in two ways: beacon mode and non beacon mode.

In the non beacon mode, transceiver only listens to the channel if there is free channel it sends the data otherwise waits a random time slot to send its data.

But in beacon mode [15] transceiver uses super-frame form to data sending. It means that all devices wake up when the superframe is coming and after, they had received the data they will go to sleep again. In this way, a lot of energy can be saved, because the devices must not be in active mode all the time.

\section{PROPOSED TPCM}

Generally, radios in wireless communication system require a fixed transmission power for all communication and consume node's battery power according to it. In this article, we propose a mechanism TPCM, to dynamically adjust the required transmission power (Tx) of radio to save node's battery power according to LQI. For this we use PHY 802.15.4 radio that provides the value of LQI and set $\mathrm{Tx}$ at it. Radio in PHY 802.15.4 uses a DEFAULT and fixed Tx of $3 \mathrm{dBm}$ for each transmission by each node. The value of LQI between sensor nodes shows that how much a node away from other node, means the distance between sensor nodes can be estimated based on LQI.

\subsection{Proposed Mechanism}

We assume that if LQI value is high then we can minimize Tx and if value is less then we can maximize Tx. For this we assume four values of LQI and four values of Tx according to these LQI values. The TPCM for dynamically set $\mathrm{Tx}$ is described as:

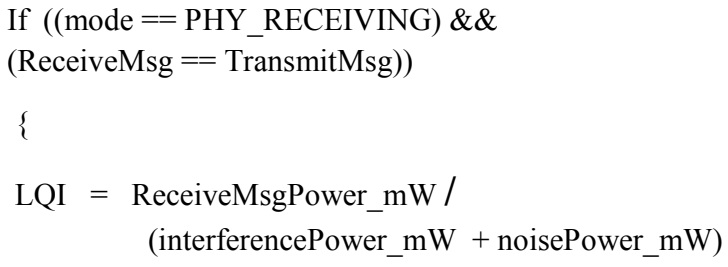




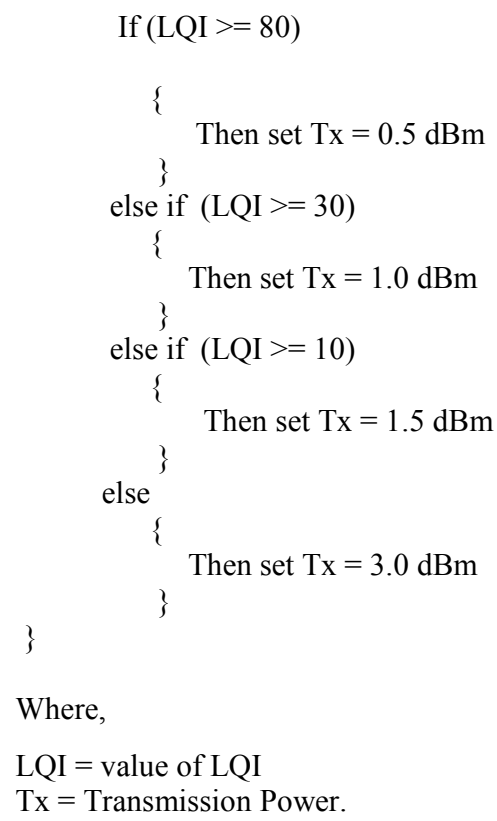

\section{PERFORMANCE METRICS}

4.1 Average End to End Delay End-to-end delay indicates how long it took for a packet to travel from the CBR source to the application layer of the destination. It represents the average data delay an application or a user experiences when transmitting data.

4.2 Throughput is the measure of the number of packets successfully transmitted to their final destination per unit time. It is the ratio between the numbers of sent packets vs. received packets.

4.3 Signal Received with error is the measure of signal received, but they have error. The error may be occurring due to noise or due to heavy traffic.

4.4 Total Bytes Received are the measure of total packet received by server. The packets may be drop due to heavy traffic. So received packets may be vary according to traffic conditions.

4.5 Average Jitter is the deviation in or displacement of some aspects of the clock pulses from the source to reach the destination with different delays. In other words it can be defined as counts the number of packets from the source will reach the destination with different delays.

4.6 Packet loss is the measure of total discarded packet due to corruption or due to packet drop. It can be calculate by subtracting total received packets by server with total sent packet by client.

4.7 Transmission Energy The transmission energy component refers to the energy consumed for transmitting packets and their associated control overhead on the radio.

\section{SIMULATION REVIEW}

All the simulations are performed in QualNet [11] wireless network simulator version 5.0. We use number of nodes 10 , Simulation time is taken 60 seconds and seed as 1 . All the scenarios have been designed in $500 \mathrm{~m} \times 500 \mathrm{~m}$ area. Mobility model used is Random Way Point (RWP). In this model a mobile node is initially placed in a random location in the simulation area, and then moved in a randomly chosen direction. The movement proceeds for a specific amount of time or distance, and the process is repeated a predetermined number of times. We simulate it with different pause time of $00 \mathrm{~s}$ to $60 \mathrm{~s}$ with AODV routing protocol. Network traffic is provided by using CBR application.

\section{RESULTS AND ANALYSIS}

In this analysis we take Pause Time versus some other parameters like End- to -End Delay, Throughput, etc. Then we compare and analyze values of our mechanism TPCM scheme with old radio or Without Transmission Power Control (WTPC) scheme.

Our first analysis is shown in Figure 1. It gives the value of endto-end delay with respect to different pause time $(\mathrm{sec})$ of both WTPC and TPCM, and shows that TPCM reduces end-to-end delay than WTPC. TPCM gives minimum and constant end-toend delay from pause time $15 \mathrm{sec}$ to $60 \mathrm{sec}$ and it is better take for a packet travel from CBR source to destination.

Figure 2 shows value of average Jitter $(\mathrm{sec})$ and pause time (sec). WTPC and TPCM at different pause time value, when we increase the pause time of nodes in network, Figure 2 shows that TPCM has less average Jitter value than WTPC. Average Jitter effect signifies the Packets from the source will reach the destination with different delays. A packet's delay varies with its position in the queues of the routers along the path between source and destination and this position can vary unpredictably. And it has been seen that the Average Jitter by TPCM is better than WTPC.

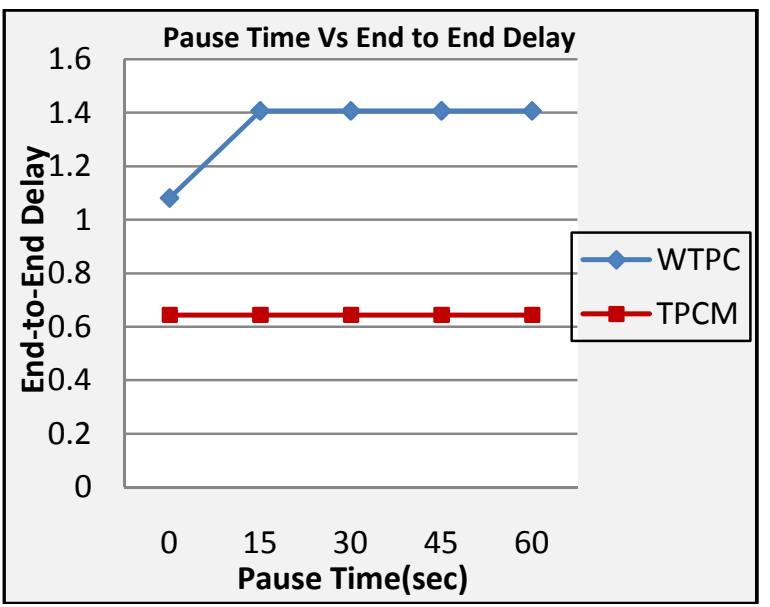

Fig. 1 


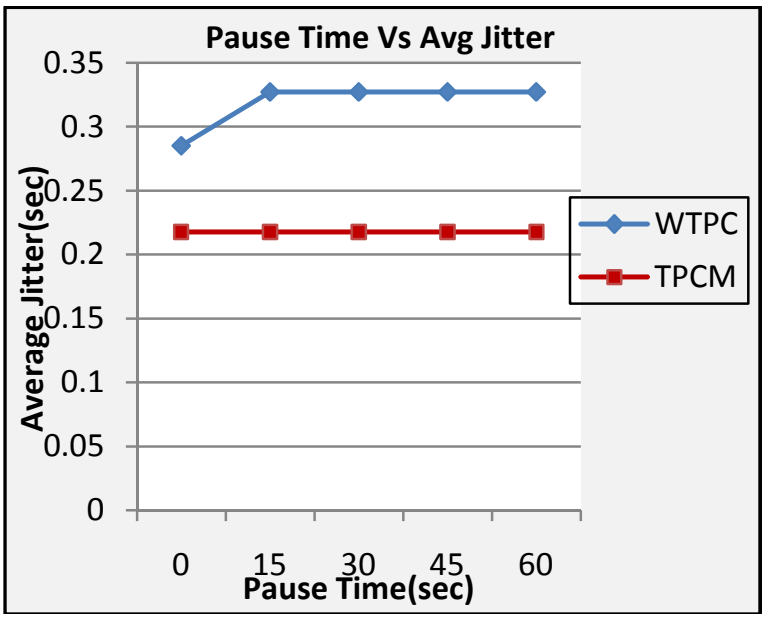

Fig. 2

Figure 3 shows total byte received by both WTPC and TPCM with variation in pause time. In TPCM, the value of total byte received is minimum at pause time $0 \mathrm{sec}$ and gives same value at other value of pause time is 40 as WTPC. We can say that TPCM has low value only at pause time $0 \mathrm{sec}$ than WTPC and at other pause time it has same performance like WTPC.

Figure 4 shows the value of Throughput (bits/s) and it also shows that at pause time $0 \mathrm{sec}$ WTPC has greater value than TPCM at pause time $0 \mathrm{sec}$. Throughput is the ratio of numbers of sent packets with number of received packets. Given figure also shows that TPCM has all values better than WTPC at different pause time excluding pause time $0 \mathrm{sec}$. It has seen that when we increase pause time the performance of WTPC is decreased and performance of TPCM is increased.

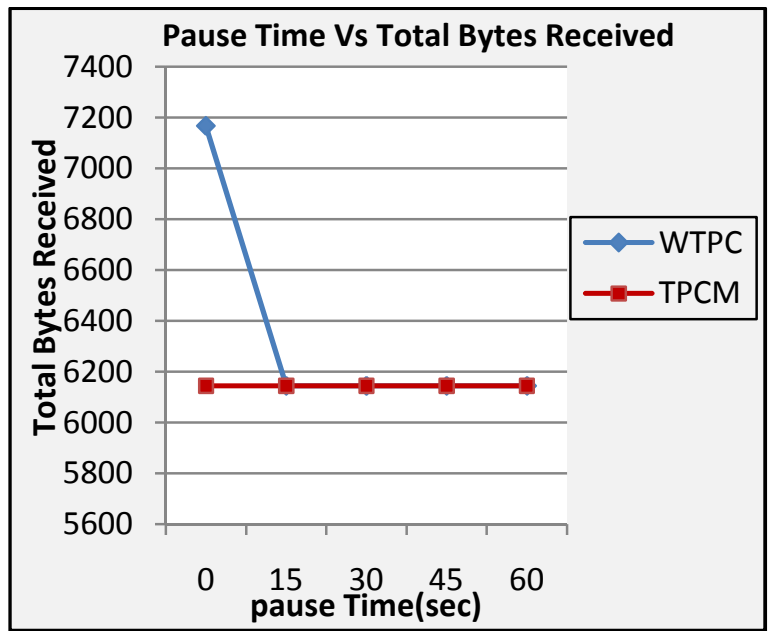

Fig.3

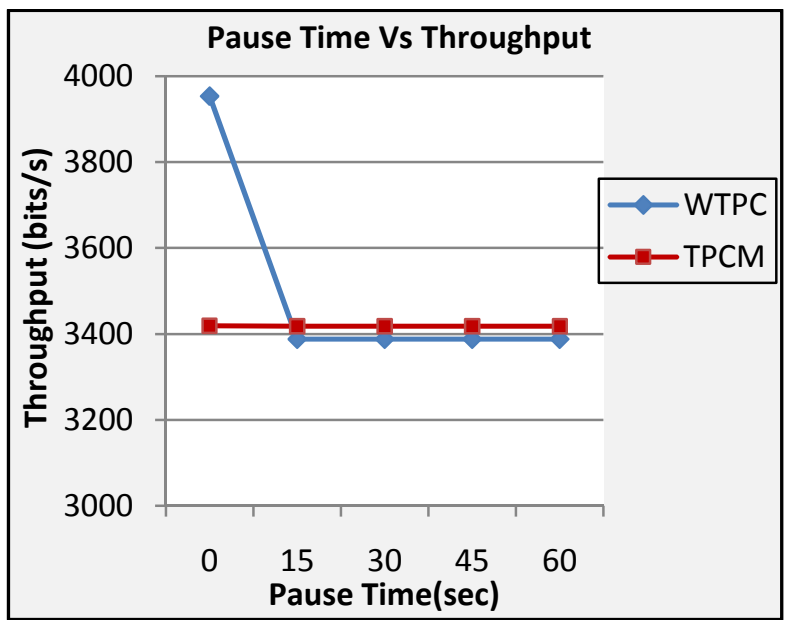

Fig.4

Figure 5 shows total packets received by both WTPC and TPCM with variation in pause time. Given figure shows that TPCM has all values same as WTPC at different pause time excluding pause time 0 sec. At pause time a sec TPCM less packet received than WTPC. It has seen that when we increase pause time the performance of WTPC is decreased but performance of TPCM is constant.

Total Packets dropped by all nodes at different pause times are shown by in figure 6 and it shows that TPCM has less packet drop during transmission than WTPC. As we can seen that at pause time 0 sec both have high value of packet loss or drop because at pause time $0 \mathrm{sec}$ the mobility of nodes is high and at high mobility there are more change of route break, due to route break there can be more packet drop. It has been seen in figure 6 that when pause time is increased the value of packet drop is decreased. As we can see that TPCM has better performance than WTPC at all values of pause time because of one reason that TPCM reduced the path break due to power conservation in TPCM.

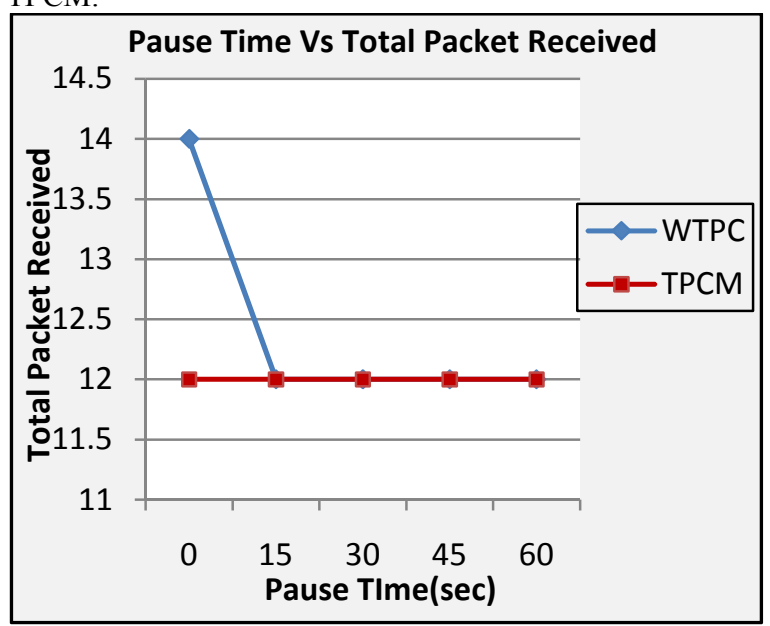

Fig.5 


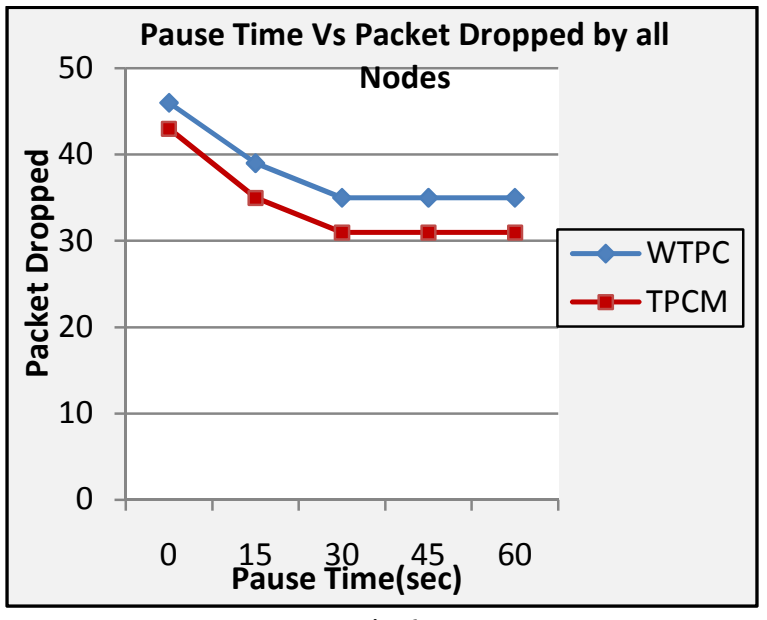

Fig.6

Value of total signal detected by all nodes of both networks of both WTPC and TPCM is shown in Figure 7, and it gives an analysis that at each value of pause time TPCM gives a better performance than WTPC means nodes in network of TPCM detect more signal than nodes in network of WTPC. Figure 7 also shows that TPCM has better performance than WTPC at all pause time. TPCM gives better performance because its power saving technique that saves the power of each nodes, it makes nodes as well as networks lifetime long, if any node exists more in network it can detects more signal in network, it is the reason of good performance of TPCM.

Figure 8 gives the value of signal received with error. Nodes at physical layer receive signal from other nodes, some time these signals received by nodes have error due to signal strength or congestion. Figure 8 gives the value of total signal with error in both networks WTPC and TPCM with varying in pause time and it also shows the effect of different pause time on these networks. At pause time $0 \mathrm{sec}$, as we can see from given values, both WTPC and TPCM receive same numbers of signal with error. When we increase value of pause time the value of error signals is increased in WTPC and reduced in TPCM. We can analyze from above result that TPCM has better performance than WTPC.

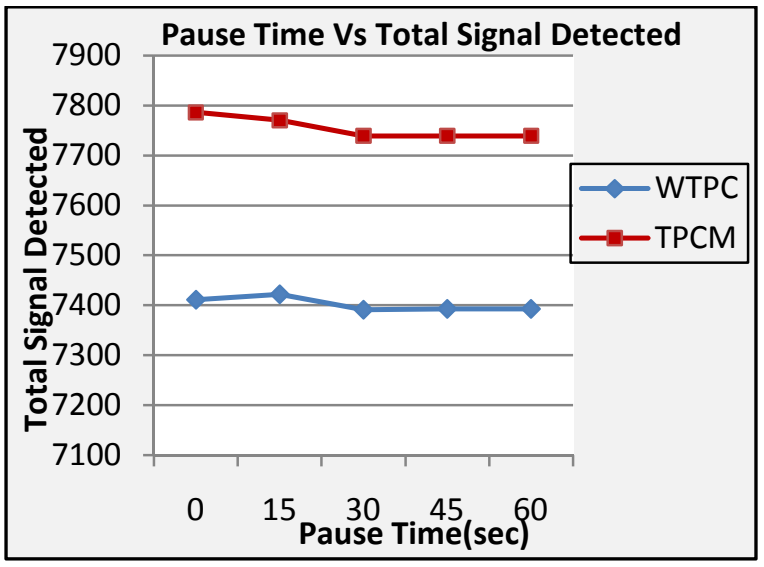

Fig.7

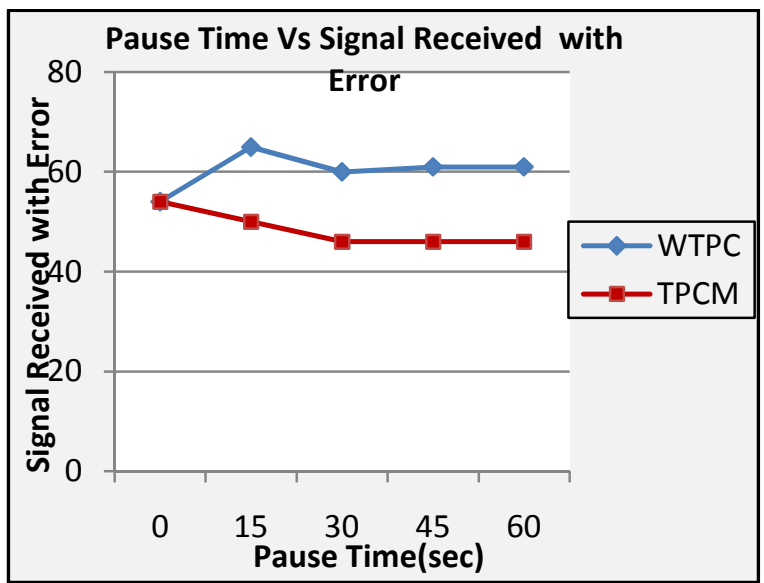

Fig. 8

The value of total signal locked by all nodes at PHY layer is given by figure 9 . It is observed that nodes in TPCM locks more signals than nodes at WTPC.

Figure 10 shows total signal received and forwarded to MAC, and gives that TPCM receives and forwards more signals to MAC than WTPC. In communication system, physical layer of one network receives signals from other network and process it. This is after process send to upper layer means MAC layer of network. We can analyze from given figure that PHY of TPCM receives and forward more signals to MAC than WTPC at different values of pause time. It can also be seen in figure 10 that value of signals received and forward to MAC is increased from pause time $0 \mathrm{sec}$ to $15 \mathrm{sec}$ and then constant at increasing value of pause time.

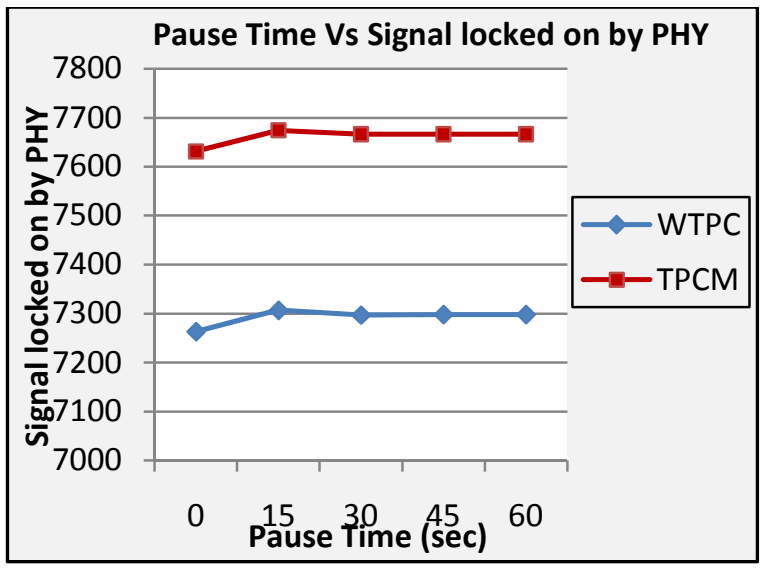

Fig.9 


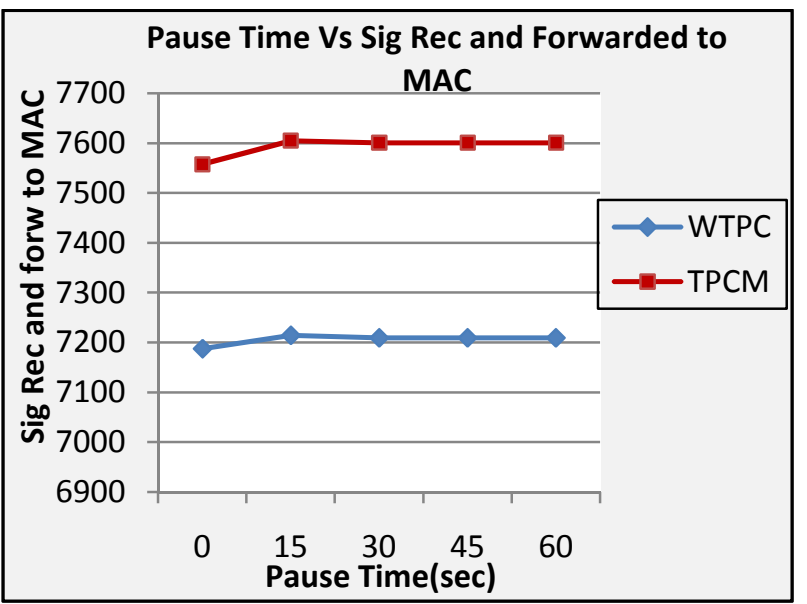

Fig. 10

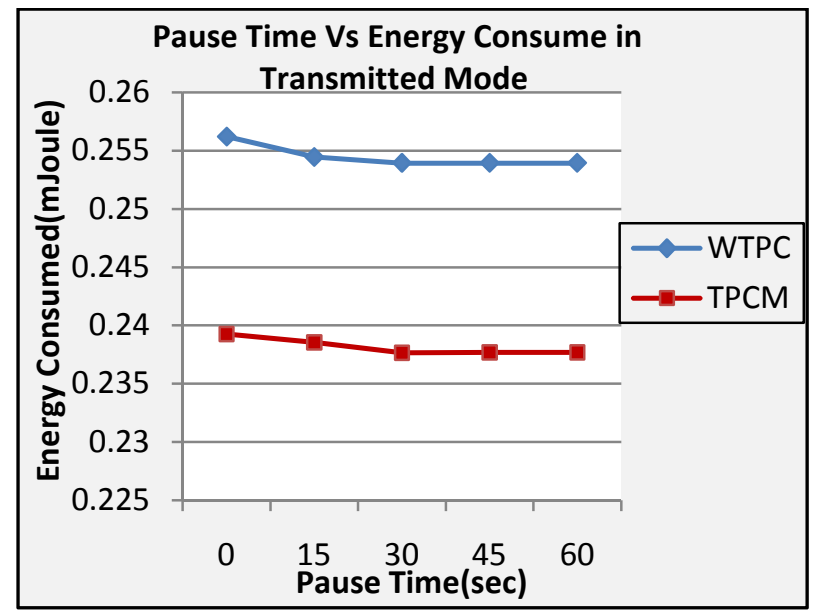

Fig.11

TPCM is design on basis of dynamically set of transmission power of radio PHY 802.15.4. Figure 11 shows values of energy consumed by all nodes of both network of WTPC and TPCM at different pause time. This Figure also gives an analysis that total energy consumed by all nodes of TPCM is less than total energy consumed by all nodes of WTPC. Energy consumption by all nodes of TPCM at all different values of pause time is less than energy consumption by all nodes of WTPC. It can be given by this result that TPCM is better than WTPC.

\section{CONCLUSION}

All the result and observations is concluded that our proposed Transmission Power Control Management (TPCM) scheme gives overall good performance than With Transmission Power Control (WTPC) scheme. At some application layer parameters it gives some poor performance at only pause time $00 \mathrm{Sec}$.

\section{FUTURE WORK}

In this paper we proposed a scheme TPCM to control the transmission power of radio. It can also be happened in this work that one can find the distance between the nodes based on value of LQI and then dynamically set the value of transmission power based on distance

\section{REFERENCES}

[1] I.Akyildz,W. Su,Y.Sankarasubramaniam and E. Cayirci ,“A survey on sensor networks ", IEEE Commu. Magazine, vol. 40, no. 8, Aug. 2002, pp. $102-114$.

[2] Nikolaos A. Pantazis and Dimitrios D. Vergados "A Survey on Power Control Issues in WIreless Sensor Networks" IEEE Communications Surveys \& Tutorials - 4th Quarter 2007, pp. 86-107.

[3] Murali P, Challa A, Kasyap M R and Chittaranjan Hota” A Generalized Energy Consumption Model for Wireless Sensor Networks" IEEE International Conference on Computational Intelligence and Communication Systems 2010, pp.210-213.

[4] Yangfan Zhou and Michael R. Lyu and Jiangchuan Liu "On Setting up Energy-Efficient Paths with Transmitter Power Controlling Wireless Sensor Networks” IEEE, 2005.

[5] Ankit Thakkar and Dr. S.N. Pradhan" Power Aware Scheduling for Adhoc Sensor Network Nodes” IEEE, 2009.

[6] Raja Jurdak, Antonio G. Ruzzelli, and Gregory M.P. O’Hare "Radio Sleep Mode Optimization in Wireless Sensor Networks", IEEE Transactions on Mobile Computing, vol. 9, no. 7, July 2010, pp. 955-968.

[7] Kun Yang, Yuan-ming WU, Hai-bo Zhou "Research of Optimal Energy Consumption Model in Wireless Sensor Network" IEEE, volume 7, 2010, pp. 421-424.

[8] Saoucene Mahfoudh, Pascale Minet "Survey of energy efficient strategies in wireless ad hoc and sensor networks" IEEE Seventh International Conference on Networking, 2008, pp.1-7.

[9] Yong-Zheng Li, Lei Wang, Xiao-Ming Wu, and Yuan-Ting Zhang "Experimental Analysis on Radio Transmission and Localization of a Zigbee-based Wireless Healthcare Monitoring Platform" IEEE 5th International Conference on Information Technology and Application in Biomedicine, May, 2008, pp.488-490.

[10] Baozhu Li and Hui Wang “A Low Complexity Localization Algorithm in Wireless Sensor Network" IEEE International Conference on Innovative Computing and Communication and Asia-Pacific Conference on Information Technology and Ocean Engineering, 2010, pp.217-220.

[11] Matthew M. Holland, Ryan G. Aures and Wendi B. Heinzelman "Experimental Investigation of Radio Performance in Wireless Sensor Networks".

[12] Juan Ignacio Huircán, Carlos Munoz, Hector Young, Ludwig Von Dossow, Jaime Bustos, Gabriel Vivallo, Marcelo Toneatti "ZigBee-based wireless sensor network localization for cattle monitoring in grazing fields" Computers and Electronics in Agriculture 74, 2010, pp. 258-264.

[13] Cherif Diallo, Michel Marot, and Monique Becker "Link Quality and Local Load Balancing Routing Mechanisms in Wireless Sensor Networks". 
[14] Cherif Diallo, Michel Marot, and Monique Becker "Using LQI to Improve Cluster Head Locations in Dense ZigBee based Wireless Sensor Networks".

[15] Paolo Baronti, Prashant Pillai, Vince Chook1, Stefano Chessa, Alberto Gotta, and Y. Fun $\mathrm{Hu}$ "Wireless Sensor Networks: a Survey on the State of the Art and the 802.15.4 and ZigBee Standards".

[16] Shabnam Pirnia "Energy Consumption in Wireless Sensor Network" November 16, 2010.
[17] Jason Lester Hill" System Architecture for Wireless Sensor Networks" PhD Report, 2003.

[18] Neeraj Choudhary, Ajay K Sharma" Performance Evaluation of LR-WPAN for different Path-Loss Models" International Journal of Computer Applications (0975 8887) Volume 7- No.10, October 2010.

[19] Scalable Network Technology, "QualNet 5.0 simulator" tutorial and QualNet Forum, http://www.scalablenetworks.com/forums.

[20] QualNet 5.0 simulator " Libraries/ Documentation ". 